\title{
Commentary: Predicting Outcomes in Patients with Asymptomatic Moderate to Severe Aortic Stenosis
}

\author{
Robert C Bahler*, Neal V Dawson \\ Case Western Reserve University School of Medicine at MetroHealth Medical Center, Cleveland, Ohio, United States of America
}

Article Info

\section{Article Notes}

Received: September 26, 2018

Accepted: October 22, 2018

\section{${ }^{*}$ Correspondence:}

Dr. Robert C Bahler, MD, 2500 MetroHealth Drive,

Cleveland, OH 44124 USA; Telephone No: 216 778-2005;

Fax No: 216 778-3927; E-mail: rbahler@metrohealth.org.

(c) 2018 Bahler RC. This article is distributed under the terms of the Creative Commons Attribution 4.0 International License.

\section{Keywords:}

Asymptomatic aortic stenosis

Left ventricular hypertrophy

Echocardiography

Diastolic dysfunction

Electrocardiography

Natural history
Abstract

Outcomes in patients with moderate to severe aortic stenosis (AS) from a community hospital cohort are similar those reported from tertiary institutions. Echocardiographic measures of the severity of obstruction are key variables in predicting outcomes. Additional variables that are not direct measures of AS severity are associated with adverse events. A reduced left ventricular (LV) ejection fraction forecasts a less favorable outcome yet most asymptomatic patients have preserved LV ejection fractions. Consequently, other measures of LV systolic function associated with adverse outcomes are important; namely, reduced LV longitudinal shortening and increased LV mass. Diastolic function can be compromised in AS indicated by either elevated E/ $\mathrm{e}^{\prime}$ or left atrial enlargement; both are associated with unfavorable outcomes. Electrocardiographic evidence of LV strain reflects LV mid-wall fibrosis and identifies patients with adverse outcomes. Biomarkers including elevated values of high sensitivity troponin I and BNP contribute to models predicting outcomes. Prediction models help identify asymptomatic patients with quite unfavorable prognoses who may benefit from early aortic valve replacement.

Decisions as to the optimal timing for aortic valve (AV) replacement in patients with aortic stenosis (AS) are of increasing importance as the population ages and $\mathrm{AV}$ replacement has become the most frequent valvular procedure. Onset of heart failure, syncope, angina and new exertional dyspnea are well established indications for proceeding with $\mathrm{AV}$ replacement since the prognosis is grave if the stenosis is not relieved. However, in asymptomatic patients with moderate or severe AS there remains uncertainty as to how well one can predict the development of symptoms or adverse outcome events. In the face of this uncertainty, there have been ongoing discussions as to whether one should intervene even in selected asymptomatic patients with severe AV obstruction. Clearly the severity of obstruction as gauged by the peak velocity across the $\mathrm{AV}$ (Vmax), the mean transvalvular gradient, the actual $\mathrm{AV}$ area and the extent of AV valve calcification are key elements in our toolbox that assist in predicting outcomes ${ }^{1,2}$. Our study demonstrated in a diverse community hospital cohort that clinical outcomes for asymptomatic patients with moderate to severe AS were primarily related to echocardiographic and electrocardiographic variables that reflected both the severity of AS and the resulting alterations of the LV myocardium and the left atrium. The discriminant power of Vmax was enhanced when these covariates were included in the outcome analyses, and co-morbidities did not enhance the prediction of outcomes in this cohort ${ }^{3}$. The rate of progression of the stenosis is also a strong predictor of outcomes ${ }^{4}$. However, apart from these direct assessments of the severity of AV stenosis, there 
are additional factors that should be considered in our efforts to predict outcomes.

How well the left ventricle (LV) responds to the markedly increased afterload affects prognosis. A reduced left ventricular ejection fraction (LVEF) is a potential harbinger of poor outcomes both short-term and longterm $^{5}$. When there is evidence of a reduced LVEF, the immediate success of valve replacement is high, yet the long-term outlook is less favorable as compared to patients undergoing valve replacement with a preserved LVEF. Can we identify LVs that are not functioning normally despite a preserved LVEF? Multiple techniques that assess longitudinal shortening of the $\mathrm{LV}$ have identified that a reduction in this variable, even with a preserved LVEF, forecasts less favorable outcomes ${ }^{6}$. Longitudinal shortening has been measured by the mitral annulus systolic excursion and, most recently, by the global longitudinal strain as measured by either echocardiography or cardiac magnetic resonance imaging ((CMR). We examined the simple and easily obtained systolic velocity ( $s^{\prime}$ ) of the mitral annulus movement towards the apex as measured by Doppler echocardiography and patients with adverse outcomes had a reduced velocity ${ }^{3}$. Longitudinal shortening is impaired in many conditions such as diabetes, hypertension and hypertrophic cardiomyopathy, and this altered function is associated with a less favorable prognosis ${ }^{7-9}$. Onset of symptoms with exercise stress testing also identifies patients in whom the LV function can no longer tolerate the added stress of exercise and therefore their risk of adverse outcomes is significantly increased ${ }^{10}$.

The hemodynamic burden faced by the LV can be described by the valvulo-arterial impedance and this parameter includes the systemic arterial systolic pressure and the mean pressure gradient across the stenotic AV. Thus, it reflects the total hemodynamic burden rather than only the abnormal afterload imposed by the severity of AS obstruction. Some authors have found this to be more closely associated with outcomes than the standard measures of AS severity ${ }^{11}$. We also found that it was associated with adverse outcomes but less strongly as compared to Vmax. The loss of arterial compliance from aging, atherosclerosis and hypertension leads to increased arterial impedance and this arterial pathology is reflected in the measurement of valvulo-arterial impedance.

Abnormal LV diastolic function is associated with unfavorable outcomes in patients with AS. This is most directly assessed by the early diastolic velocity of the mitral annulus $\left(e^{\prime}\right)$ which reflects impaired LV relaxation. When expressed as the E/ $\mathrm{e}^{\prime}$ ratio it has a strong association with adverse outcomes in patients with severe $\mathrm{AS}^{12}$. We also observed that increases in the ratio of E/ e' are associated with less favorable outcomes. Long standing LV diastolic dysfunction can result in enlargement of the left atrium and our data and that of others have shown that this enlargement is associated with adverse outcomes ${ }^{13}$.

Another imaging parameter that heralds less favorable outcomes is an abnormally increased LV mass inde ${ }^{14}$. This variable reflects the duration and severity of the continued LV systolic pressure overload and is frequently associated with impaired systolic function. Cox regression models have identified that LV mass index has a strong association with all cardiovascular events but is strongly associated with death and heart failure ${ }^{14}$.

An electrocardiogram (ECG) should always be part of the evaluation of patients with AS and we found that the T wave changes of LV strain were associated with unfavorable outcomes; an observation supported by prior observations regarding ECG abnormalities ${ }^{15,16}$. Recent CMR data have identified that the LV strain pattern is associated with LV mid-wall fibrosis ${ }^{16}$. Fibrosis reflects ongoing myocyte death and is therefore identifying LVs that are being stressed beyond their ability to maintain normal function. Increased duration of the QRS likely reflects LV myocardial damage and again is associated with poor outcomes ${ }^{17}$. Longstanding pressure overload of the LV eventually can lead to left atrial enlargement and to atrial fibrillation; both were associated with poor outcomes in our patients with $\mathrm{AS}$ and in prior publications ${ }^{18,19}$.

Although not part of our study, biomarkers have gained interest in identifying patients with AS and evidence of LV dysfunction. The BNP has been extensively studied and, when there is evidence of BNP increases, onset of symptoms is proximate ${ }^{20}$. A less well studied biomarker is high-sensitivity troponin assays. Increased levels indicate ongoing myocardial necrosis and one recent report has shown that high-sensitivity troponin I may be a very useful marker of adverse outcomes and abnormal increases are associated with myocardial fibrosis ${ }^{21}$.

Are clinical variables able to contribute to the assessment of prognosis in patients with AS? There is no dispute that increasing age is associated with more hemodynamically important AS and onset of symptoms. We found that the other demographics such as sex and BMI were not useful in assessing risk. Surprising, comorbid conditions, that clearly assess the risk associated with AV replacement, did not add incrementally to measures of association in a prediction model in our study and have not been shown to be helpful is assessing the possibility of an adverse outcomes in patients with moderate to severe $\mathrm{AS}^{2,3}$.

With this background there have been several efforts to construct risk scores to help determine who might be considered for AV replacement prior to actual symptoms. One such proposal was based on data from echocardiography and included higher Vmax, calcium score, and observed/predicted left ventricular (LV) mass ratio ${ }^{22}$. 
A recent report showed that by combining distinct types of data including 1) age, 2) sex, 3) Vmax, 4) ECG evidence of LV strain and 5) the serum level of high-sensitivity troponin I a clinical risk score could be defined that performed very well, including in a validation cohort ${ }^{23}$. The need for very robust prediction models has increased as the age of our population has increased and our knowledge that delays in $\mathrm{AV}$ replacement can adversely alter the long-term outcome of patients. A prospective study of patients with moderate to severe AS from several large health care systems could provide the necessary data to establish robust prediction models that would greatly enhance a physician's ability to recommend valve replacement in selected patients prior to the onset of clear symptoms.

\section{References}

1. Baumgartner $\mathrm{H}$, Hung J, Bermejo J, et al. Echocardiographic assessment of valve stenosis: EAE/ASE recommendations for clinical practice. Eur J Echocardiogr. 2009; 10: 1-25.

2. Bhattacharyya S, Hayward C, Pepper J, et al. Risk stratification in asymptomatic severe aortic stenosis: a critical appraisal. Eur Heart J. 2012; 33: 2377-87.

3. Bahler RC, Hawi R, Rovner A, et al. Am J Cardiol. 2018 Sep 1; 122(5): 851-858. doi: 10.1016/j.amjcard.2018.05.027. Epub 2018 Jun 4.

4. Rosenhek R, Binder T, Porenta G, et al. Predictors of outcomes I severe, asymptomatic aortic stenosis. N Engl J Med. 2000; 343: 611-7.

5. Barbash IM, Minha S, Ben-Dor I, et al. Relation of preprocedural assessment of myocardial contractility reserve on outcomes of aortic stenosis patients with impaired left ventricular function undergoing transcatheter aortic valve implantation. Am J Cardiol. 2014; 113: 1536-1542.

6. Fries B, Liu D, Gaudron P, et al. Role of global longitudinal strain in the prediction of outcome in patients with severe aortic valve stenosis. Am J Cardiol. 2017; 120: 640-647.

7. Kouzu H, Yuda S, Muranaka A, et al. Left ventricular hypertrophy causes different changes in longitudinal, radial, and circumferential mechanics in patients with hypertension: a two-dimensional speckle tracking study. J Am Soc Echocardiogr. 2011; 24(2): 192-199.

8. Enomoto M, Ishizu T, Seo Y, et al. Myocardial dysfunction identified by three-dimensional speckle tracking echocardiography in type 2diabetes patients relates to complications of microangiopathy. J Cardiol. 2016; 68(4): 282-287.

9. Urbano-Moral JA, Rowin EJ, Maron MS, et al. Investigation of global and regional myocardial mechanics with 3-dimensional speckle tracking echocardiography and relations to hypertrophy and fibrosis in hypertrophic cardiomyopathy. Circ Cardiovasc Imaging. 2014; 7(1): 11-9.

10. Amato MCM, Moffa PI, Werner KE, et al, Treatment decisions in asymptomatic aortic valve stenosis: role of exercise testing. Heart 2001; 86: 381-386.

11. Zito C, Salvia J, Cusma-Piccione M, et al. Prognostic significance of valvuloarterial impedance and left ventricular longitudinal function in asymptomatic severe aortic stenosis involving three-cuspid valves. Am J Cardiol. 2011; 108: 1463-9.

12. Biner S, Rafique AM, Goykhman $P$, et al. Prognostic value of E/E' ratio in patients with unoperated severe aortic stenosis. J Am Coll Cardiol Img. 2010; 3: 899-907.

13. Casaclang-Verzosa G, Malouf JF, Scott CG, et al. Does left atrial size predict mortality in asymptomatic patients with severe aortic stenosis: Echocardiography 2010; 27: 105-9.

14. Gerdis E, Rossebø AB, Pedersen TR, et al. Relation of left ventricular mass to prognosis in initially asymptomatic mild to moderate aortic valve stenosis. Circ Cardiovasc Imaging. 2015; 8: e003644.

15. Greve AM, Boman K, Gohlke-Baerwolf $\mathrm{C}$, et al. Clinical implications of electrocardiographic left ventricular strain and hypertrophy in asymptomatic patients with aortic stenosis: the Simvastatin and Ezetimibe in Aortic Stenosis study. Circulation. 2012; 125: 346-53.

16. Shah AS, Chin CW, Vassiliou V, et al. Left ventricular hypertrophy and strain and aortic stenosis. Circulation. 2014; 130: 1607-16.

17. Greve AM. Risk stratifying asymptomatic aortic stenosis: role of the resting 12-lead ECG. Dan Med J. 2014; 61: B4793.

18. Christensen NL, Dahl JS, Carter-Storch R, et al. Relation of left atrial size, cardiac morphology, and clinical outcome in asymptomatic aortic stenosis. Am J Cardiol. 2017; 120: 1877-1883.

19. Levy F, Rusinaru D, Maréchaux S, et al. Determinants and prognosis of atrial fibrillation in patients with aortic stenosis. Am J Cardiol. 2015; 116: $1541-1546$.

20. Lancellotti P, Moonen M, Magne J, et al. Prognostic effect of long-axis left ventricular dysfunction and B-type natriuretic peptide levels in asymptomatic aortic stenosis. Am J Cardiol. 2010; 105: 383-8.

21. Dahou A, Clavel MA, Capolade R, et al. B-Type Natriuretic Peptide and High-Sensitivity Cardiac Troponin for Risk Stratification in Low-Flow, Low-Gradient Aortic Stenosis: A Substudy of the TOPAS Study. JACC Cardiovasc Imaging. 2018; 11: 939-947.

22. Cioffi G, Mazzone C, Faggiano P, et al. Prognostic stratification by conventional echocardiography of patients with aortic stenosis: the “CAIMAN-ECHO score”. Echocardiography. 2013; 30: 367-77.

23. Chin CWL, Messika-Zeitoun D, Shah AS, et al. A clinical risk score of myocardial fibrosis predicts adverse outcomes in aortic stenosis. Eur Heart J. 2016; 37: 713-723. 